\title{
The use of over-the-counter medication by elderly medical in-patients
}

\author{
Gwenno M Batty, C Alice Oborne, CG Swift, SHD Jackson
}

\begin{abstract}
Summary
Use of over-the-counter (OTC) medications by elderly patients is often not identified. This survey was performed to study the use of OTCs by medical inpatients aged 65 and over. Data on the use of OTC medications before and during hospital admission were collected by questioning patients and case notes were examined for documentation of their use of OTC medications. OTC medications were used by 44 of 138 (32\%) patients interviewed. Patients used a total of 70 OTC medications before admission and six OTC medications were being used during hospital admission. There was no documentation of pre-admission and inhospital OTC medicine use in the clinical notes and patients had little knowledge of the potential harm some products can cause. As more products become available over the counter, doctors should record their use in patients' notes and patients should be encouraged to seek professional advice before purchasing OTC medicines and to read the product information leaflets.
\end{abstract}

Keywords: over-the-counter medications, elderly

Elderly Medical Unit, Queen Mary's Hospital, Sidcup, Kent DA14 6LT, UK GM Batty

Clinical Age Research Unit, Department of Health Care of the Elderly, King's College School of Medicine and Dentistry, London SE5 9PJ, UK CA Oborne CG Swift SHD Jackson

Correspondence to Gwenno M Batty, Department of Health Care of the Elderly, Care of the Elderly,
Clinical Age Research Clinical Age Research Unit, King's College Dentistry, Bessemer Road, London SE5 9PJ, UK

Accepted 1 May 1997 hospital physicians take accurate histories of medication use and frequently fail to identify the use of OTC drugs in medication his- tories. ${ }^{9,10}$ Doctors dealing with elderly patients should be particularly aware of possible OTC medicine use, as elderly patients have a higher incidence of adverse drug reactions. ${ }^{11}$ A study from the US showed that up to $96 \%$ of patients of all ages surveyed used OTCs and aspirin was the most commonly used drug. ${ }^{12}$ A further survey of community patients aged 75 years and over in the UK found almost half the patients admitted to taking non-prescribed medicines. ${ }^{13}$ Elderly patients have been shown to take OTC analgesics more often, for longer periods and in combination with a greater number of prescribed medicines, ${ }^{14}$ and a further study found just over a third of the OTCs used by elderly people in the community were analgesics or aspirin. ${ }^{15}$

To our knowledge, there are no data on OTC use by medical in-patients. This survey was performed to study the use of OTC medicines by elderly patients before and during hospital admission.

\section{Methods}

All patients aged 65 years and over on medical wards at two hospitals, were questioned on their use of OTC medicines in the preceding year. Reasons for exclusion from further questioning included dysphasia, dysarthria, dementia, and disorientation. Patients were asked to look at the OTC Directory of the Proprietary Association of Great Britain to assist recognition of OTC medicines. Patients using OTC medicines before admission were questioned regarding place of purchase of OTC medicine, regularity of use, duration of use, and use of OTC medicine during current admission. Patients were also questioned on the indications and contraindications of the OTC medicines used.

Medical case notes and nursing card indexes of patients using OTC medicines were examined for documentation of use of OTC medicines, reason for admission and possible contraindication to the OTC medicine. Drug charts of patients using OTC medicines were examined for prescribing of drugs during inpatient stay which had previously been purchased over the counter by patients. Research Ethics Committee approval was granted.

\section{Results}

Comprehensive drug histories on use of OTCs were collected in 138 of 190 patients interviewed. The remaining 52 patients were unable 


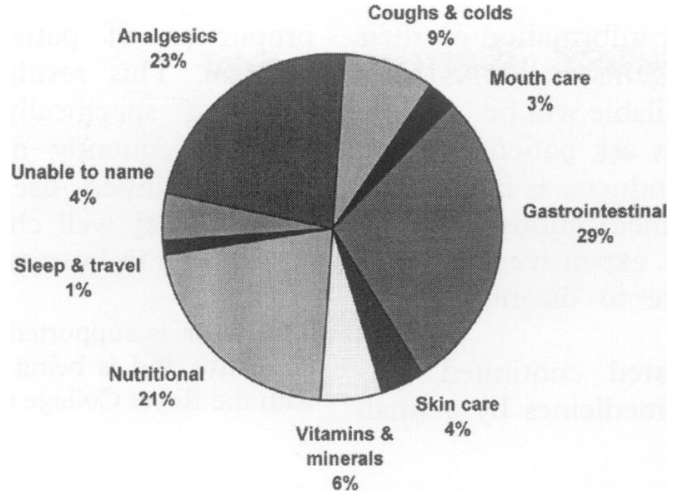

Figure OTC medicines (a total of 70 items) used by 44 patients out of 138 hospital in-patients aged 65 years and over

to participate and were excluded from further interview. A total of $44(32 \%)$ patients were found to be taking at least one OTC preparation and used a total of 70 OTC medicines. Gastrointestinal products accounted for over a quarter of medications used (figure). Analgesics, which have the potential to cause significant adverse effects, were also frequently used, nine patients taking aspirin and one taking ibuprofen. Cod liver oil, used by 10 patients, was the commonest single preparation. Three patients were unable to recall the name or indication for the OTC preparation they were using. The number of preparations used by patients varied, with one ( 28 patients) and two (13 patients) being most common, but a single patient took seven products.

Products had been purchased from a community pharmacy in 59 cases and from supermarkets or health food shops in 11 cases. This would theoretically have enabled a community pharmacist to advise the majority of patients on the appropriateness of their purchase. Patients claimed to have read the product information leaflet for $16(23 \%)$ products and demonstrated knowledge of the indication for therapy for $47(67 \%)$ products. Knowledge of contraindications and adverse effects of OTC medicine used was demonstrated for only six drugs. No patient case notes contained documentation of pre-admission use of OTC medicines, in spite of patients using preparations daily and for long duration. OTC medicines were used daily by 18 patients and 52 medicines had been used for many years. Examination of patients' drug charts revealed five drugs taken before admission as OTC medicines were prescribed for patients whilst in hospital and six OTC medicines were being used by patients in hospital without documentation of awareness by medical staff. One patient was using her own supply of OTC 'Anadin' whilst an inpatient and was prescribed aspirin.

The pre-admission use of an OTC medicine was duplicating the medication prescribed by a general practitioner in one patient. The patient was prescribed aspirin $75 \mathrm{mg}$ and was taking OTC aspirin $300 \mathrm{mg}$, three times a day. The patient also used 'Rennies', purchased OTC, for indigestion. It was felt that OTCs contributed towards admission in two patients.
One patient admitted with a haemorrhagic stroke was taking two aspirin tablets of unknown strength, up to three times a day for headaches. A further patient admitted with gout was taking low dose aspirin.

\section{Discussion}

This is the first study to examine the use of OTC medicines by in-patients aged 65 years and over before and during hospital admission. We found $32 \%$ of patients interviewed were using OTC medicines. Studies from the US ${ }^{16}$ and Australia ${ }^{17}$ have reported much higher proportions of non-institutionalised elderly patients use OTC medicines. The discrepancy between our findings and such studies could be explained by the differing systems of healthcare. In the UK, patients must be offered the choice of an National Health Service prescription $^{18}$ and as prescriptions are free for women aged 60 years or over, and recently men of the same age, many elderly patients may chose to have drugs prescribed for financial reasons. Studies from the US have demonstrated that many elderly patients attempt symptom relief by self-prescription before seeking professional advice $^{19}$ and purchase OTC medicines under the direction of a physician, ${ }^{20}$ in an effort to save money. The setting for data collection could also account for the lower prevalence of OTC medicine use in our study. Domiciliary data collections were performed in the US ${ }^{16}$ and UK studies, ${ }^{13}$ which would encourage patients to search their homes for OTC medicines. Also, the demography, case-mix and inclusion criteria of the different studies could explain the variation. Our sample derived from urban areas and one Australian study has shown rural elderly patients use more OTC medicines than urban elderly patients. ${ }^{21}$ In addition, our patients were in hospital and therefore more ill than patients in the community not requiring hospital admission. Our iller patients are more likely to have seen a doctor and are therefore potentially less likely to selfmedicate.

Although the majority of patients knew why they were using OTC medicines, few patients had read the product information leaflet or were familiar with adverse effects of the constituents. Also, elderly patients may be less likely to read product information leaflets as they are frequently unable to read the small printed material on OTC medications. ${ }^{22}$

Despite prolonged and frequent use of OTC medicines, there was no documentation of selfprescribed medications in patients' case notes.

\begin{tabular}{|l|}
\hline Summary/learning points \\
\hline - patients do not volunteer information on use \\
of OTCs \\
- documentation of pre-admission OTC use in \\
clinical notes did not occur \\
- patients continue to use OTCs in hospital \\
of OTCnts are not familiar with the adverse effects \\
of OTed
\end{tabular}


Patients do not volunteer information on their use of non-prescription agents, believing that a drug which is readily available will be 'safe'. It is important that doctors ask patients if they have been using OTC products, as incomplete documentation of all medications in drug histories can result in expensive, needless investigations and failure to diagnose druginduced illnesses.

Our study demonstrated continued selfadministration of OTC medicines by a small

1 Anonymous. Mrs Bottomley set on curbing drug bill. Pharm 7 1992; 249: 748 .

2 Department of Health. The health of the nation. London: HMSO, 1992.

3 Kennedy JG. Over the counter drugs: changing the roles of doctors and pharmacists. $B M \mathcal{F}$ 1996; 312: 593-4.

4 Ayres JG, Fleming DM, Whittington RM. Asthma death due to ibuprofen. Lancet 1987; 1: 1082 .

5 Scarpinato L, Purdom D. Theophylline toxic effects from an over-the-counter asthma medication. Arch Fam Med 1993; 2: $1081-3$.

6 Stricht BIV, Parvais OE, van Haelen Fastre RJ, van Haelen MH. Remedies may contain cocktail of active drugs. $B M \mathcal{Y}$ 1994. Remedies may

7 Caranasos GJ, Stewart RB, Cluff LE. Drug-induced illness leading to hospitalisation. $\mathscr{f} A M A$ 1974; 228: $713-7$.

8 Henry JA. Hazards of self-medication. Br F Clin Pract 1994 48: 285.

9 Cann PA. Nonsteroidal anti-inflammatory drugs and peptic ulcers. $B M F$ 1990; 300: 875-6.

10 Beers $\mathrm{MH}$, Munekata MS, Storrie M. The accuracy of medication histories in the hospital medical records of elderly persons. F Am Geriatr Soc 1990; 38: 1183-7.

11 Hurwitz N. Predisposing factors in adverse reactions to drugs. $B M F$ 1969; 1: 536-9.

12 Darnell JC, Murray MD, Martz BL, Weinberger $M$ Medication use by ambulatory elderly: an in-home survey. f Am Geriatr Soc 1986; 34: 1-4. proportion of patients after admission to hospital. This resulted from physicians not inquiring specifically about self-medication and consequently not advising patients on their continued use. Having spent money, patients may well choose to take their medicines, even in hospital.

This work is supported by the NHS Management Executive and is being conducted in collaboration with the Royal College of Physicians Research Unit.

13 Blenkiron, P. The elderly and their medication: under standing and compliance in a family practice. Postgrad Med 996; 72: $671-6$

14 Whitaker P, Wilson R, Bargh J, Chapman M, Dudley R. Use and misuse of purchased analgesics with age. Pharm $\mathcal{f}$ 1995; 254: $553-6$.

15 Cartwright A, Smith C. Over-the-counter medicines. In: Elderly people, their medicines, and their doctors. London: Routledge, 1988.

16 Guttmann D. Patterns of legal drug use by older Americans. Addict Dis Int f 1978; 3: $337-56$.

17 Simons LA, Tett S, Simons J, et al. Multiple medication use in the elderly: use of prescription and non-prescription drugs in an Australian community setting. Med $\mathscr{f}$ Aust 1992 157: $242-6$.

18 Department of Health Press Release 1993. Ref: H93/1092.

19 Gerbino PP, Gans JA. Antacids and laxatives for symptomatic relief in the elderly. $\mathcal{F}$ Am Geriatr Soc 1982; 30: S81 S87.

20 May FE, Stewart RB, Hale WE, Marks RG. Prescribed and nonprescribed drug use in an ambulatory elderly population. South Med f 1982; 75: $522-8$.

21 Moore JF, Johnson JE. Over-the-counter drug use by the rural elderly. Geriatr Nurs 1993; 14: 190-1.

22 Watanabe RK, Gilbreath K, Sakamoto CC. The ability of the geriatric population to read labels on over-the-counter medication containers. $¥$ Am Optom Assoc 1994; 65: 32-7. 